$1 \quad$ Evolutionary causes of lifespan extension by dietary restriction: linking

\title{
theory and mechanisms
}

4 Laura M. Travers*, Hanne Carlsson, Elizabeth M. L. Duxbury, and Alexei A. Maklakov

5

6

7

8

9

10

11

12

13

14

15

16

17

18

19

20

21 Keywords: Ageing, Autophagy, Dietary Restriction, Life-History Theory, Senescence 


\section{Abstract}

Dietary restriction (DR), reduced food intake without malnutrition, increases lifespan across a broad range of taxa, but the evolutionary underpinning of this phenomenon is poorly understood. The resource reallocation hypothesis proposes that dietary restricted animals divert resources from reproduction to somatic maintenance to increase survival in times of nutrient scarcity in favour of future reproduction. The "longevity by-product" hypothesis proposes instead that dietary restricted animals increase nutrient recycling via autophagy to maximise immediate reproduction, thereby reducing cellular toxic waste and leading to longer lifespan as an unselected by-product. The "longevity by-product" hypothesis makes a unique prediction that blocking autophagy in DR animals will simultaneously reduce lifespan and reproduction. To test the adaptive value of autophagy under dietary restriction, we inhibited autophagy using bec-1 RNAi knockdown in DR and fully-fed Caenorhabditis elegans nematodes. Our findings confirm that autophagic inhibition results in a significantly shorter lifespan under DR, suggesting that autophagy is important for survival in times of famine. Remarkably, we also show that inhibiting autophagy throughout adult life significantly increases reproduction in both dietary restricted and fully fed worms. Moreover, this did not come at a transgenerational cost to offspring fitness. Our results suggest that autophagy is an energetically costly process that reduces resources available for reproduction, but is necessary for survival during famine, and are thus consistent with the resource reallocation hypothesis. 
Dietary restriction (DR), a moderate reduction in food intake without malnutrition, extends lifespan in many organisms, from yeast to primates ${ }^{1-4}$. DR is the most reproducible environmental intervention to increase lifespan and improve health outcomes in a wide range of species ${ }^{5-7}$. Research on genes that modulate lifespan has revealed that nutrient-sensing pathways play a key role in the physiological changes that affect ageing and lifespan in response to nutrient availability ${ }^{8-10}$. Changes in activation of nutrient-sensing pathways in response to nutrient availability are thought to reflect an adaptive phenotypically plastic response, allowing organisms to optimise investment between somatic maintenance and reproduction to maximise fitness in varying environments ${ }^{11,12}$. The predominant evolutionary theory, the resource reallocation hypothesis, proposes that the physiological effect of DR, characterised by increased lifespan and reduced reproduction is an adaptive strategy to increase survival in times of resource scarcity ${ }^{12}$. By diverting resources away from reproduction and towards somatic maintenance, organisms can maximise their chances of survival by reducing investment into energetically costly activities such as reproduction, and prioritise getting through temporary famine by investing in somatic maintenance. According to this theory, when nutrients become plentiful again, organisms can reverse the allocation from somatic maintenance, back into reproduction ${ }^{12}$.

However, several shortcomings of the resource reallocation theory have recently been highlighted. While DR commonly results in a decrease in reproduction, recent studies have shown that reduced reproduction is not a requisite for increased lifespan (for a review, see ${ }^{13,14}$ ). For example, DR can extend lifespan in sterile and non-reproducing animals ${ }^{3,15-17}$, which suggests that lifespan extension may occur in the absence of resource allocation from reproduction to somatic maintenance. Experimental evolution in D. melanogaster has also

67 found no evidence for coevolution between longevity and reproduction under DR ${ }^{18,19}$. These 
findings highlight that extended lifespan under DR may not be constrained by direct competition for limited resources as suggested by the resource reallocation theory.

In addition to the evidence disputing the mechanistic underpinnings of resource reallocation, Adler \& Bonduriansky ${ }^{20}$ challenge the evolutionary explanation of the adaptive resource reallocation theory, that is, that the DR response functions to increase survival over reproduction. One of their key criticisms is that increasing investment into somatic maintenance to promote survival, while significantly decreasing or "shutting down" reproduction, is not adaptive. Given that selection should favour reproduction over longevity, they argue that delaying reproduction during food shortages would not be adaptive in the wild where extrinsic mortality is high for many species ${ }^{20}$. In summary, the evidence above casts doubt on whether dietary restricted animals strategically allocate resources between somatic maintenance and reproduction to improve survival in times of famine.

Adler \& Bonduriansky ${ }^{20}$ propose an alternative evolutionary explanation for the DR response by focusing on the physiological cell protection and repair mechanisms that are triggered in response to DR. One key cellular process involved in removing and recycling damaged cell components that is upregulated in response to DR is autophagy. Autophagy is a process whereby portions of the cytoplasm, including misfolded proteins, mitochondria, and other organelles, are degraded, catabolized and recycled ${ }^{21}$. Large double-membrane vesicles called autophagosomes are generated, which engulf cytoplasmic organelles. The autophagosomes fuse with lysosomes where the breakdown products are recycled to the cytoplasm. Adler \& Bonduriansky ${ }^{20}$ argue that the primary evolutionary function of increased autophagy under DR is not to improve somatic maintenance to maximise survival under starvation, but instead to free up nutrients through increased nutrient recycling to invest in reproduction. If delaying reproduction is not adaptive due to the risk of extrinsic mortality, upregulating autophagy would allow the organism to make the most efficient use of scarce 
resources for some immediate reproduction. In this scenario, extended lifespan in response to

DR is merely a non-adaptive by-product of increased autophagy. According to this theory, increased autophagy is beneficial by making more efficient use of resources when nutrients are scarce, which results in the availability of more nutrients for reproduction that would otherwise not be available ${ }^{20}$.

The evolutionary question of why selection has favoured the up- and downregulation of autophagy in response to nutrient availability remains unanswered. We aimed to test whether the primary role of increased autophagy, one of the key mechanisms involved in extending lifespan under $\mathrm{DR}^{22-28}$, is to increase survival as suggested by the resource reallocation theory ${ }^{12}$, or to increase immediate reproduction as proposed by the longevity by-product hypothesis ${ }^{20}$. The longevity by-product hypothesis posits that increased autophagy frees up resources for reproduction. Therefore, this theory makes the unique prediction that reproduction should decrease if autophagy is inhibited in DR conditions because the extra nutrients normally made available through upregulated autophagy would not be available to invest in reproduction here. In this study, we tested the adaptive significance of increased autophagy under DR. We used RNA interference (RNAi) to inhibit a key autophagy gene in dietary restricted and ad lib fed Caenorhabditis elegans worms, and examined the consequences for reproduction and lifespan. We also tested for potential transgenerational consequences of inhibiting autophagy in fully fed worms by measuring reproduction and lifespan in the offspring of ad lib fed parents.

\section{Methods}

\section{Strains}

We used Bristol N2 wild type and eat-2 (ad 1116) mutant (genetic model of DR) strains of C. elegans nematodes in all assays. Eat-2 mutants have a defect in a nicotinic acetylcholine 
receptor subunit that functions in the pharyngeal muscle ${ }^{29}$, resulting in reduced pharyngeal pumping rates and $20 \%$ less food intake ${ }^{30}$. Due to dietary restriction, eat-2 mutants live approximately $20 \%$ longer and have fewer offspring $(\sim 50 \%)$ than wild type worms ${ }^{15,27}$.

Before the start of the experiment, we bleached plates to collect eggs from worms recovered from frozen. We used standard NGM agar plates to grow the nematode populations ${ }^{31}$, with added antibiotics $(100 \mu \mathrm{g} / \mathrm{ml}$ Ampicillin and $100 \mu \mathrm{g} / \mathrm{ml}$ Streptomycin) and a fungicide (10 $\mu \mathrm{g} / \mathrm{ml}$ Nystatin) to avoid infections ${ }^{32}$. Before the experiment began, we fed the nematode populations antibiotic resistant Escherichia coli OP50-1 (pUC4K), gifted by J. Ewbank at the Centre d'Immunologie de Marseille-Luminy, France. From defrosting and throughout the experiment, we kept worms in $20^{\circ} \mathrm{C}$ and $60 \%$ relative humidity climate chambers. We used RNAi to knock down Beclin-1, an ortholog of mammalian Beclin1, a gene that is part of an operon that contains the stress-inducible transcription factor gene $s k n-1$ which is essential for the lifespan extension caused by DR ${ }^{33}$. RNAi bec- 1 was provided by the Julie Ahringer RNAi library. Previous studies have shown inactivation of bec-1 in eat-2 mutants abrogates the lifespan extension effects of DR $22,26,27$. Thus, BEC-1 is required for the lifespan extension induced by dietary restriction in eat-2. While inhibiting bec-1 prevents food limitation from extending lifespan, it has also been shown to have little effect on the lifespan of well-fed animals ${ }^{27}$.

In order to induce RNAi knockdown, we fed the nematodes strain HT115 (DE3) of E. coli containing a Timmons and Fire feeding vector L4440 modified to express the dsRNA of bec1. In addition to the RNAi knockdown bacteria, we used a strain of HT115 carrying an empty L4440 feeding vector to feed to worms in our control treatment ${ }^{34}$. We grew cultures of the RNAi clones in LB medium supplemented with Ampicillin $(50 \mu \mathrm{g} / \mathrm{ml})$ and then seeded onto $35 \mathrm{~mm}$ standard NGM plates with the addition of IPTG $(1 \mathrm{mM})$ and Ampicillin $(50 \mu \mathrm{g} / \mathrm{ml})$, as recommended by Kamath et al. ${ }^{35}$. After seeding plates with either RNAi bec-1 knockdown or 
the control empty vector RNAi, we grew the bacteria overnight in $20^{\circ} \mathrm{C}$ to induce expression of RNAi, before placing worms on the plates.

\section{Experimental design and set-up}

For logistical reasons, we conducted the lifespan assay in three experimental blocks and the reproduction assay in four blocks. We collected age-synchronized eggs from N2 and eat-2 worms at peak reproduction (Day 2 in N2 and Day 3 in eat-2) from unmated (i.e. self-fertilised) hermaphrodites fed on OP50-1 (pUC4K). Eggs from each strain were placed on OP50-1 plates to develop from egg to late L4/Day 1 adults. We placed eggs onto the development plates, which we assigned a random identifier (e.g. 1-10) to blind the experimenter to the worm strain (N2 and eat-2) henceforth.

At the beginning of the experiment, age-synchronised (Day 1) adult worms from both strains were randomly selected from the development plates and placed on either RNAi bec- 1 seeded plates or on empty vector control plates and maintained on their respective plate type throughout life. We used a combination of randomisation and stratification to set-up and handle experimental plates throughout the experiment, with plates assigned random identifiers (e.g. 1-100). Thus, experimenters were blind to worm treatment and strain during the daily transfers when we scored lifespan, and during offspring counting.

\section{Offspring production}

To obtain data on the number of offspring produced by each worm, we reared single unmated (i.e. self-fertilised) hermaphrodites on individual plates from adult Day 1 until reproduction ceased. We moved the worms onto new plates every $24 \mathrm{~h}$, incubated eggs for $48 \mathrm{hrs}$ at $20^{\circ} \mathrm{C}$, and counted the number of progeny. For each block, 15-30 worms were set up for each treatment-strain combination. Over the four blocks, we assayed a total of 69-85 worms per treatment-strain combination. 
Lifespan assays were performed on unmated hermaphrodites from adult Day 1 until death. We set up worms in groups of ten and transferred onto fresh plates daily while scoring survival.

Death was defined as the absence of movement in response to touch. For each block, we set up 50-100 worms for each treatment-strain combination. Over the three blocks, we assayed a total of 170 worms per treatment-strain combination.

Offspring reproduction and lifespan assays

To explore the transgenerational consequences of inhibiting autophagy in parents, we measured progeny production and lifespan in the offspring of fully fed $\mathrm{N} 2$ worms exposed to RNAi bec1 in adulthood. We collected age-synchronized eggs from RNAi bec- 1 and control RNAi empty vector fed N2 worms at peak reproduction (Day 2). At the beginning of both the offspring lifespan and reproduction assay, age-synchronised (Day 1) adult worms were haphazardly selected from the development plates and placed on standard NGM agar plates seeded with $0.1 \mathrm{ml}$ of Escherichia coli OP50-1 (pUC4K) and maintained on OP50-1 plates throughout the duration of both assays. In the reproduction assay, we placed single unmated (i.e. self-fertilised) hermaphrodites on individual plates from adult Day 1 until reproduction ceased. For the lifespan assay, we set up worms in groups of ten and transferred onto fresh plates daily while scoring survival. Both assays were conducted in two experimental blocks. As with the parental assays, offspring assays were conducted randomised and blinded, as described above.

We used R version 3.5.1 ${ }^{36}$ for all statistical analyses and figures. We analysed lifetime reproductive success (LRS; total number of offspring produced per individual) in both the parents and offspring assays using linear mixed effects models (LMM) implemented in the 
interaction as fixed effects, along with the development plate (plate on which the individual worm developed from egg to adult prior to the beginning of the experiment) nested within experimental block as random intercepts. For offspring LRS, we fitted treatment and block (centred) as fixed effects, as well as the development plate nested within experimental block as random intercepts. We obtained P values from LMMs from t-tests using Satterthwaite's approximation for denominator degrees of freedom implemented in lmerTest $^{38}$. We also analysed LRS by bootstrapping using the dabestr package ${ }^{39}$. The $95 \%$ confidence intervals are graphically represented by the package in Figures 1(a) (parents) and 3(a) (offspring).

We analysed lifespan in both parents and offspring using mixed effects Cox proportional hazard models implemented in the coxme package version 2.2-14 ${ }^{40}$. Individuals that died of matricide (internal hatching of eggs) or escaped from agar were censored. For parental lifespan we fitted treatment and strain (both centred), and their interaction as fixed effects. We centred the treatment and strain by coding factor levels as minus 0.5 and 0.5 , respectively, to facilitate the interpretation of main effects in the presence of an interaction ${ }^{41}$. We included random intercepts for experimental blocks and group plate to account for variation due to shared environments. For offspring lifespan, we fitted treatment and block (centered) as fixed effects (offspring were all from the N2 strain) along with group plate as a random intercept.

\section{Results}

We found a significant effect of treatment (RNAi bec-1 and empty vector control) on lifetime reproduction in both the eat-2 dietary restricted mutants and $\mathrm{N} 2$ wild type ad lib fed worms. Eat-2 and N2 worms that were fed RNAi bec-1 produced on average $19 \%$ and $11 \%$ more offspring, respectively, compared to control worms fed the empty vector $\left(\mathrm{t}_{1,250.5}=5.69, \mathrm{p}<\right.$ 0.001: Figure 1 and Table 1). There was no significant interaction between treatment and nematode ( $\mathrm{N} 2$ and eat-2) strain $\left(\mathrm{t}_{1,248.9}=-0.83, \mathrm{p}=0.406\right)$, hence we removed the interaction 
from the final model. As expected, eat-2 worms produced fewer offspring (mean $=144 \pm 5.30$

$\mathrm{SE})$ than the wild type $\mathrm{N} 2($ mean $=248 \pm 7.04 \mathrm{SE} ; \mathrm{t} 1,61.9=-17.14, \mathrm{p}<0.001)$.

We found a marginally non-significant interaction between treatment and strain for lifespan (z $=1.94, \mathrm{df}=1, \mathrm{p}<0.053)$. Inhibition of autophagy reduced lifespan in DR worms substantially more than in N2 worms (proportional hazard odds ratios 2.06 versus 1.3). This means DR worms had a two-fold increase in mortality risk when we inhibited autophagy, while autophagy inhibition increased mortality in N2 worms only by a third (Figure 2 and Table S1).

We found no effect of parental inhibition of autophagy on either LRS $\left(t_{1,93.4}=-0.94, p=0.349\right.$ : in the offspring of fully fed $\mathrm{N} 2$ worms.

\section{Discussion}

We tested the adaptive significance of increased autophagy in response to nutrient scarcity. For many years, it had been assumed that autophagy acts to promote survival by maintaining cellular homeostasis during periods of famine ${ }^{42}$. A more recent suggestion is that autophagy may be activated during famine to free up resources for immediate reproduction ${ }^{20}$. A unique prediction of this "longevity by-product" hypothesis is that blocking autophagy under DR should reduce reproduction compared to DR controls with intact autophagy. We inhibited the autophagy gene bec-1, previously shown to be required for lifespan extension under DR ${ }^{22-27}$, and examined the consequences for reproduction and lifespan in DR and fully fed worms. Contrary to the prediction of the "longevity by-product" hypothesis, we found a substantial increase of $10-20 \%$ in offspring number when autophagy was inhibited, both in DR and in fully fed worms. Moreover, we confirmed previous findings that RNAi bec-1 significantly shortens lifespan in eat-2 (DR) mutants but has a lesser effect on lifespan in N2 wild type worms ${ }^{26,27}$. Our findings support the adaptive explanation proposed by the resource 
reallocation hypothesis, which posits that increased autophagy under DR is adaptive to increase survival during famine.

In this study, we aimed to test why selection has favoured the up- and downregulation of autophagy in response to nutrient availability. While our findings support the evolutionary explanation proposed by the resource reallocation theory, the mechanistic underpinnings of lifespan extension under DR remain unclear. Recent research suggests that reproduction and lifespan are not regulated through direct competition for resources, but are mediated through molecular signals ${ }^{1,14,43-46}$. When an animal is fully fed, the activation of nutrient-sensing pathways inhibits cellular recycling and repair mechanisms. Inhibited autophagy allows cell proliferation and growth, allowing an organism to increase reproduction ${ }^{1}$. Nutrient-sensing pathways are deactivated in response to nutrient scarcity, which results in the upregulation of cellular recycling mechanisms ${ }^{2}$. Thus, while increased autophagy extends lifespan through repair of damaged cells, it also limits cellular growth and proliferation ${ }^{47,48}$, which limits reproduction. We found that inhibition of autophagy resulted in an increase in reproduction in both DR and fully fed worms, suggesting that increased cell growth and proliferation resulting from decreased autophagy in both DR and wild type worms allows individuals to increase their reproduction. Thus, increased autophagy under nutrient scarcity does not serve to increase reproduction, instead showing the opposite effect. Autophagy may use up more energy than it produces, which could explain why we found an increase in reproduction when autophagy was suppressed in both DR and wild type worms.

Inhibition of other autophagy genes also shortens lifespan in DR and nutrient-sensingdeficient animals. Similar to the effects of bec-1 inhibition, suppression of atg 7 reduces lifespan in eat-2 mutants, but has little effect on the lifespan of $\mathrm{N} 2$ wild type worms ${ }^{26}$. Inhibition of bec-1, atg-7 and atg-12 reduces longevity in long-lived daf-2 C. elegans mutants ${ }^{24,25}$. Toth et al. ${ }^{22}$ also found that mutants with reduced insulin/IGF-1 signalling and dietary- 
restricted worms require genes involved in autophagy to live long. Collectively, these findings suggest that autophagy plays a key role in regulating lifespan in response to nutrients.

A key question emerging from our findings is why basal levels of autophagy are higher than what seems optimal for maximising reproduction in fully fed control worms. Increased reproduction when autophagy is inhibited in wild type worms suggests that lower expression of bec-1 should be beneficial for offspring production while incurring no costs to survival when worms are fully fed. So why do worms not plastically decrease the rate of autophagy (expression of bec-l) during times of plenty to increase their reproductive output? The important role of BEC-1 in various biological processes may shed light on why expression is not downregulated to maximise reproduction in full feeding. In C. elegans, BEC-1 is required for embryo development and viability, dauer formation and germline formation ${ }^{25,49-53}$. Given the important role of BEC-1 during development, the lack of downregulation may be an example of sub-optimal gene expression in adulthood due to the diminishing strength of selection with age ${ }^{54-56}$.

BEC-1 is also important for DNA repair; bec-1 null and bec-1 RNAi fed animals show increased accumulation of DNA damage and an increase in germline cell death due to a delay in apoptotic cell corpse degradation ${ }^{49,57}$. C. elegans with defects in DNA damage repair display a reduction in the number of viable eggs laid after DNA damage ${ }^{58}$. Therefore, while downregulation of autophagy increases the number of offspring produced, it could result in decreased offspring quality due to diminished ability to maintain the germline. However, our investigation of lifetime reproduction and lifespan in the offspring of fully fed wild type bec-1 fed parents did not reveal any transgenerational costs of parental autophagy inhibition.

\section{Conclusions}


In summary, our findings suggest that autophagy is increased in response to nutrient scarcity

287

to improve survival rather than to maximise reproduction. Therefore, they support the resource reallocation theory for why DR extends lifespan, and refute the idea that autophagy frees up resources to maximise reproduction during famine. Increased autophagy activated by decreased insulin signalling limits cell proliferation, which could trade-off with cell repair and processes that require cell proliferation such as reproduction.

Our findings suggest that reproduction could be increased with little cost to survival if autophagy is downregulated during full feeding in adulthood. The fact that fully fed wild type worms do not decrease autophagy despite the apparent potential for increasing reproductive fitness points towards hidden costs of autophagy inhibition. Future studies could further explore the impact of inhibiting parental autophagy on offspring quality by measuring the fitness consequences in a variety of stressful environments.

\section{Author contributions}

LMT and AAM conceived and designed the study. LMT, HC, and EMLD collected the data. LMT analysed the data and wrote the first draft of the manuscript. AAM revised and edited the manuscript, and all authors approved the final version.

\section{Acknowledgements}

Tom van Baalen and Kris Sales assisted with laboratory work. We thank Andreas Sutter for advice on statistical analyses. This work has been supported by ERC Consolidator Grant GermlineAgeingSoma to AAM. 
Figure 1 (a): Inhibition of autophagy increases reproduction in N2 and eat-2 worms.
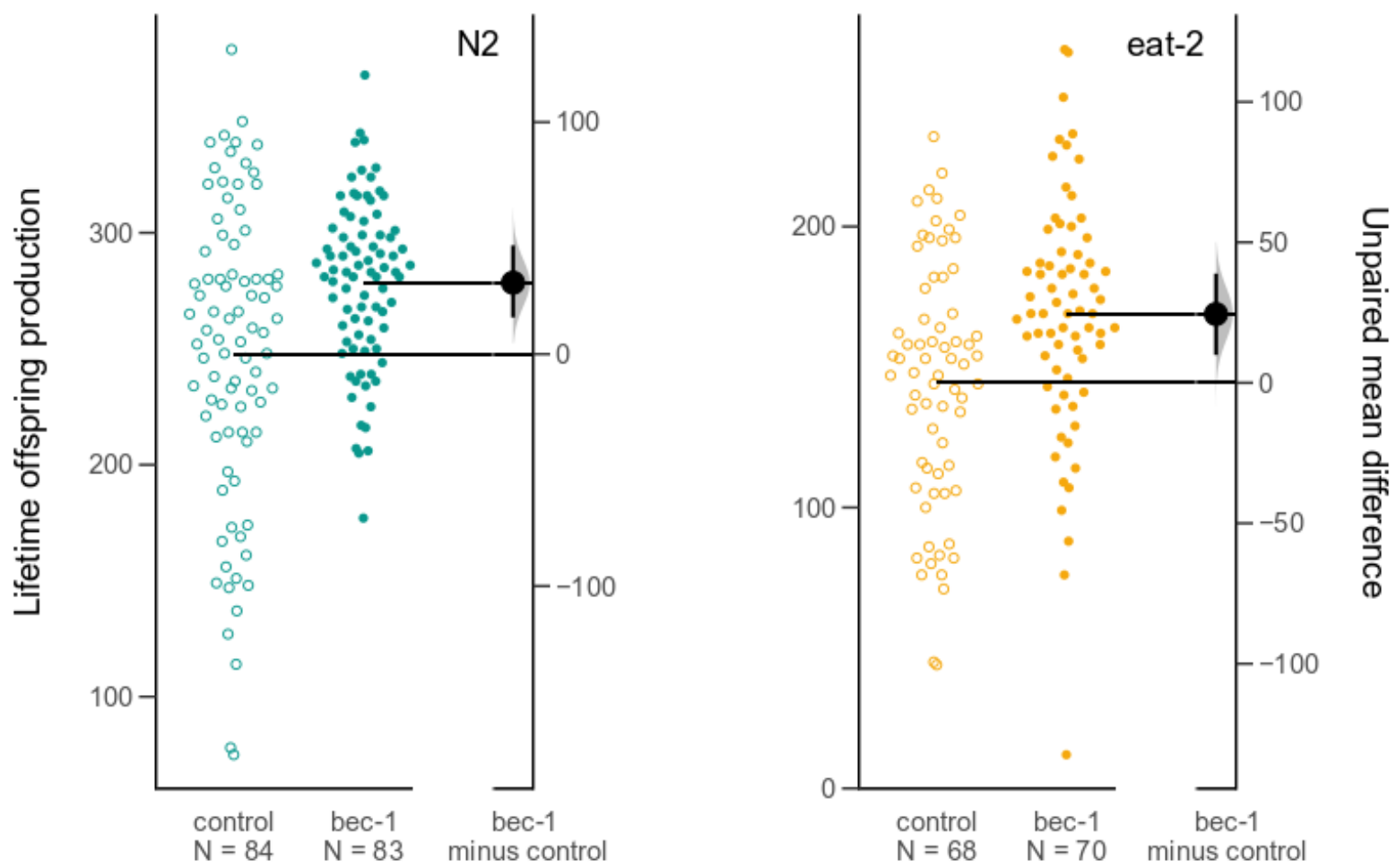

Estimation plots of total lifetime reproduction for $N 2$ (green) and eat-2 (yellow) where RNAi bec-1 treatment (filled circles) are compared to the control (open circles), with a graded sampling distribution of bootstrapped values and bootstrapped $95 \%$ CI, implemented in dabestr ${ }^{39}$. 


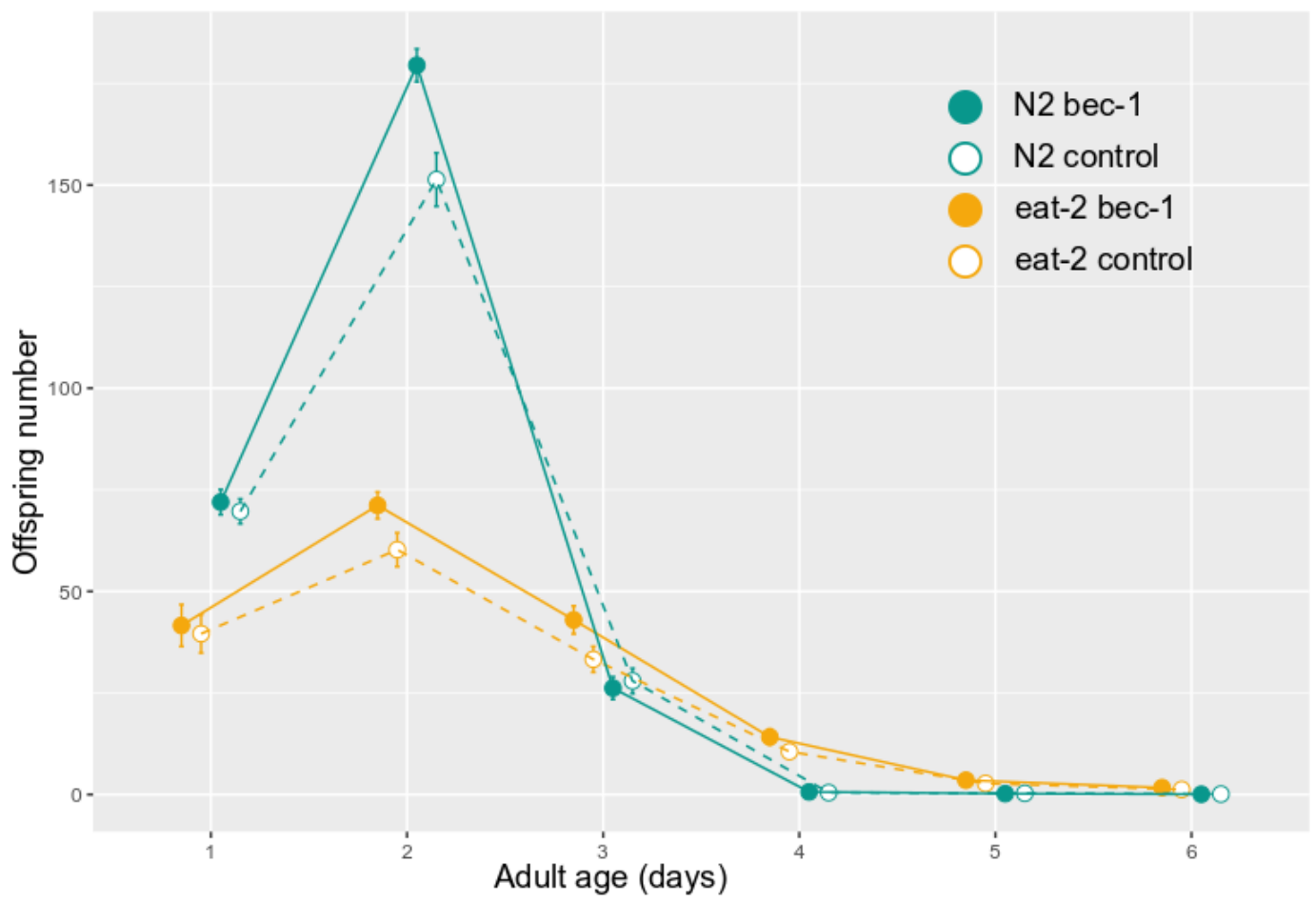

Age-specific reproduction for each nematode strain and RNAi treatment combination; circles and error bars represent mean \pm SE's. Colours indicate N2 (green) and eat-2 (yellow) worm strains. Open circles and dashed lines depict offspring number from control empty vector treatment, filled circles and solid lines depict RNAi bec-1 treatment. 
Figure 2: Reduced survival in eat-2 worms when autophagy is inhibited.

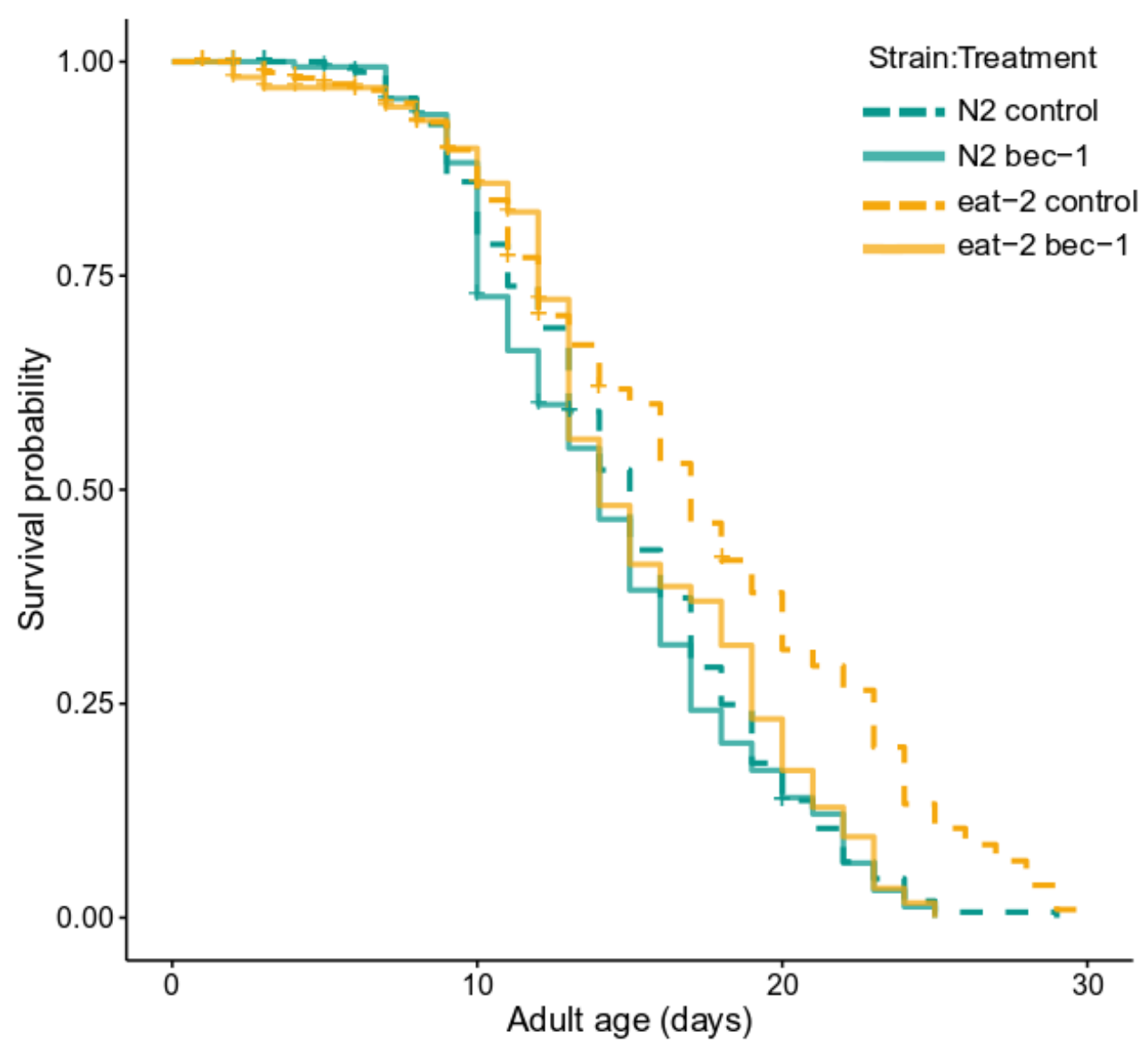

346 Survival curves for each nematode strain and RNAi treatment combination. Colours indicate $347 \quad$ N2 (green) and eat-2 (yellow) worm strains. Solid lines depict RNAi bec-1 treatment, dashed 348 lines depict control empty vector treatment. Plus-signs indicate censored individuals 349 (matricide or escape; see main text). 
Figure 3(a): No difference in reproduction in offspring of N2 parents when autophagy is inhibited compared to controls.

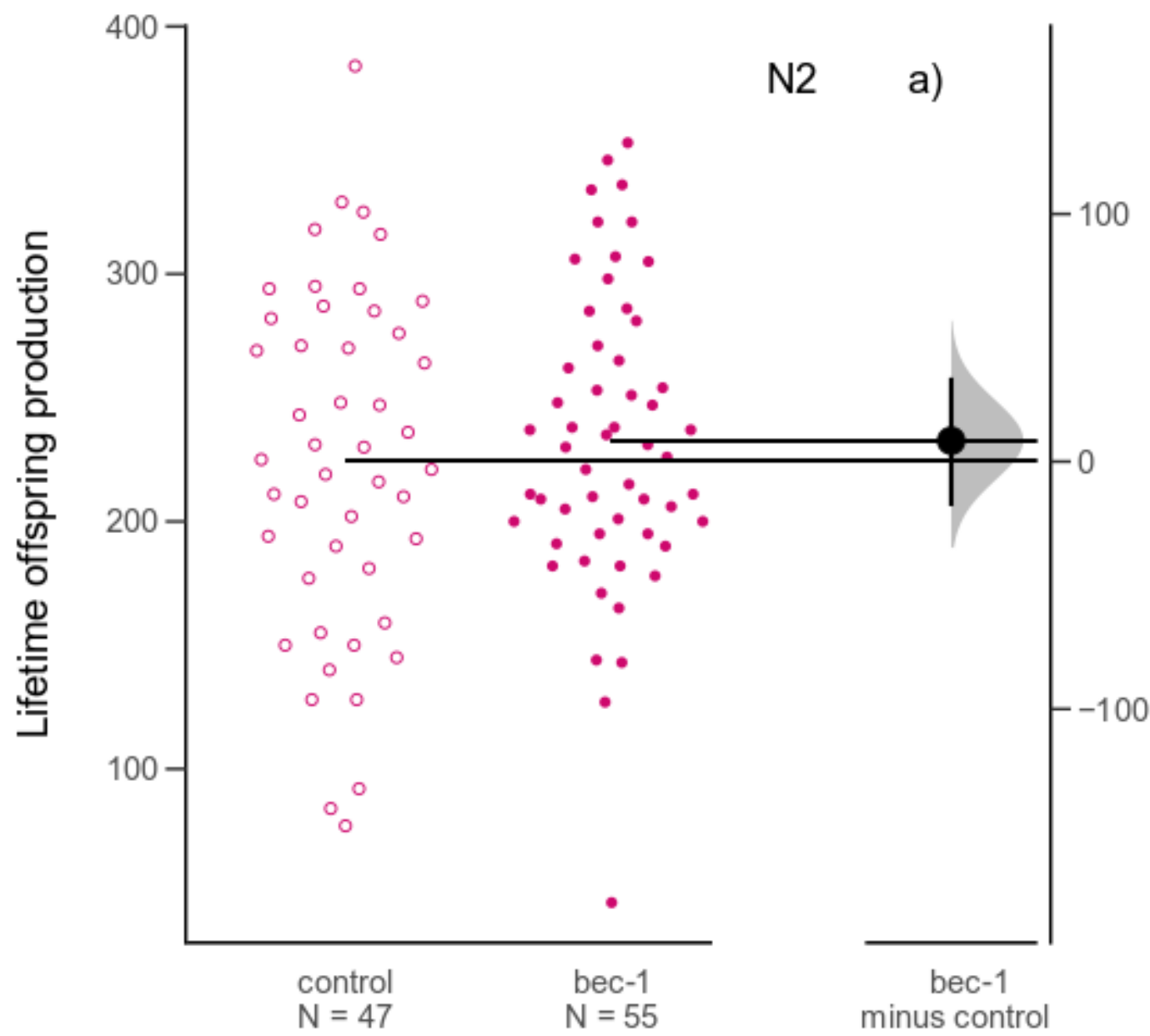


Figure 3 (b): No difference in lifespan in offspring of N2 parents when autophagy is inhibited compared to controls. Survival curves for N2 offspring.

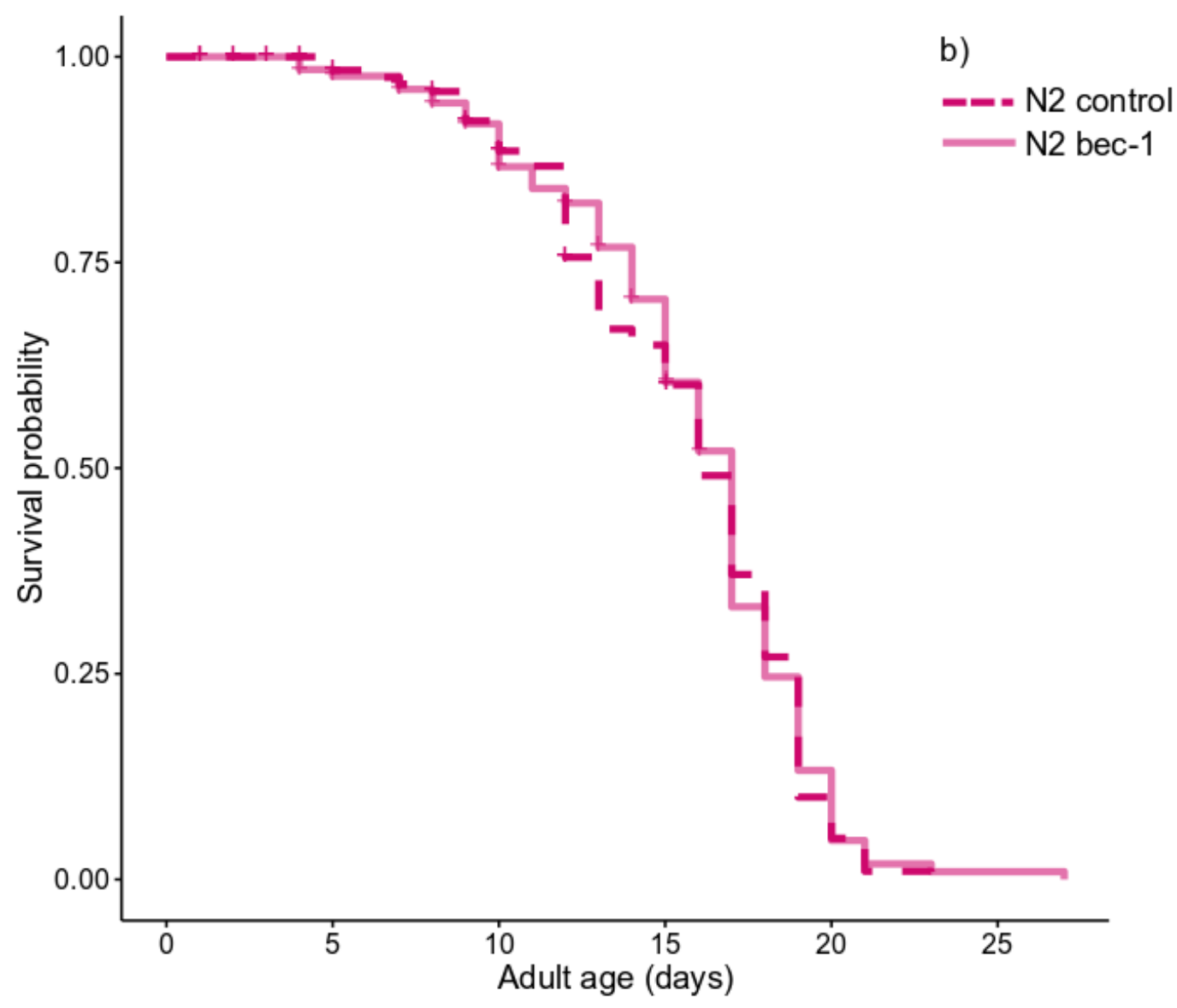
from control empty vector treated N2 worms. Plus-signs indicate censored individuals (matricide or escape; see main text). 
Table 1: Full model summary for lifetime reproduction.

Lifetime reproduction $(N=305)$

linear mixed effect model

\begin{tabular}{lccccc} 
Fixed effects & Coef & $\begin{array}{c}\text { SE } \\
\text { (coef) }\end{array}$ & ddf & $\mathbf{t}$ & $\mathbf{p}$ \\
\hline Intercept & $\mathbf{2 5 2 . 6 0 7}$ & $\mathbf{1 1 . 6 2 8}$ & $\mathbf{3 . 7 8 3}$ & $\mathbf{2 1 . 7 2 4}$ & $<\mathbf{0 . 0 0 1}$ \\
treatment & $\mathbf{- 1 0 6 . 2 6}$ & $\mathbf{6 . 2}$ & $\mathbf{2 5 0 . 5 3 6}$ & $\mathbf{5 . 6 8 7}$ & $<\mathbf{0 . 0 0 1}$ \\
strain & $\mathbf{2 7 . 7 4 6}$ & $\mathbf{4 . 8}$ & $\mathbf{6 1 . 9 8 7}$ & $\mathbf{- 1 7 . 1 4}$ & $<\mathbf{0 . 0 0 1}$ \\
& & & & & \\
Random effects & Var & SD & & & \\
\hline Block (4 levels) & 441 & 21 & & & \\
Development plate & 232.9 & 15.26 & & & \\
Residual & 1782.1 & 42.21 & & & \\
\hline
\end{tabular}

Coefficients, standard errors, test statistics and variance components are taken from a LMM on the number of offspring produced. Effects associated with a p-value smaller than 0.05 are highlighted in bold.

\section{References}

1. Kenyon, C. J. The genetics of ageing. Nature. 464, 504-512 (2010).

2. Fontana, L., Partridge, L. \& Longo, V. D. Extending healthy life span-from yeast to humans. Science. 328, 321-326 (2010).

3. Mair, W. \& Dillin, A. Aging and Survival: The Genetics of Life Span Extension by Dietary Restriction. Annu. Rev. Biochem. 77, 727-754 (2008).

4. Partridge, L., Deelen, J. \& Eline Slagboom, \& P. Facing up to the global challenges of ageing. Nature 561, 45-56 (2018).

5. Nakagawa, S., Lagisz, M., Hector, K. L. \& Spencer, H. G. Comparative and metaanalytic insights into life extension via dietary restriction. Aging Cell. 11, 401-409 (2012).

6. Liang, Y. et al. Calorie restriction is the most reasonable anti-ageing intervention: A 
meta-analysis of survival curves. Sci. Rep. 8, (2018).

402

403

404

405

406

407

408

409

410

411

412

413

414

415

416

417

418

419

420

421

422

423

7. Fontana, L. \& Partridge, L. Promoting health and longevity through diet: from model organisms to humans. Cell. 161, 106-118 (2015).

8. Slack, C., Giannakou, M. E., Foley, A., Goss, M. \& Partridge, L. dFOXO-independent effects of reduced insulin-like signaling in Drosophila. Aging Cell. 10, 735-748 (2011).

9. Zandveld, J., van den Heuvel, J., Zwaan, B. J. \& Piper, M. D. W. Both overlapping and independent mechanisms determine how diet and insulin-ligand knockouts extend lifespan of Drosophila melanogaster. Aging Mech. Dis. 3, (2017).

10. Emran, S., Yang, M., He, X., Zandveld, J. \& Piper, M. D. W. Target of rapamycin signalling mediates the lifespan-extending effects of dietary restriction by essential amino acid alteration. Aging. 6, 390-398 (2014).

11. Regan, J. C., Froy, H., Walling, C. A., Moatt, J. P. \& Nussey, D. H. Dietary restriction and insulin-like signalling pathways as adaptive plasticity: A synthesis and reevaluation. Funct. Ecol. (2019). doi:10.1111/1365-2435.13418

12. Shanley, D. P. \& Kirkwood, T. B. L. Calorie restriction and aging: A life-history analysis. Evolution. 54, 740-750 (2000).

13. Barnes, A. I. \& Partridge, L. Costing reproduction. Animal Behaviour. 66, 199-204 (2003).

14. Leroi, A. M. Molecular signals versus the Loi de Balancement. Trends in Ecology and Evolution. 16, 24-29 (2001).

15. Crawford, D., Libina, N. \& Kenyon, C. Caenorhabditis elegans integrates food and reproductive signals in lifespan determination. Aging Cell. 6, 715-721 (2007). 
16. Bjedov, I. et al. Mechanisms of life span extension by rapamycin in the fruit fly Drosophila melanogaster. Cell Metab. 11, 35-46 (2010).

17. Mitteldorf, J. Can experiments on caloric restriction be reconciled with the disposable soma theory for the evolution of senescence? Evolution. 55, 1902-6 (2001).

18. Zajitschek, F. et al. Evolution under dietary restriction decouples survival from fecundity in Drosophila melanogaster females. J. Gerontol. A. Biol. Sci. Med. Sci. 74, $1542-1548$ (2019).

19. Zajitschek, F. et al. Evolution under dietary restriction increases male reproductive performance without survival cost. Proc. R. Soc. B Biol. Sci. 283, (2015).

20. Adler, M. I. \& Bonduriansky, R. Why do the well-fed appear to die young?: A new evolutionary hypothesis for the effect of dietary restriction on lifespan. BioEssays. 36, 439-450 (2014).

21. Klionsky, D. J. \& Emr, S. D. Autophagy as a regulated pathway of cellular degradation. Science. 290, 1717-1721 (2000).

22. Tóth, M. L. et al. Longevity pathways converge on autophagy genes to regulate life span in Caenorhabditis elegans. Autophagy. 4, 330-338 (2008).

23. Hansen, M., Rubinsztein, D. C. \& Walker, D. W. Autophagy as a promoter of longevity: insights from model organisms. Nat. Rev. Mol. Cell Biol. 19, 579-593 (2018).

24. Hars, E. S. et al. Autophagy regulates ageing in C. elegans. Autophagy 3, 93-95 (2007).

25. Meléndez, A. et al. Autophagy genes are essential for dauer development and life-span extension in C. elegans. Science. 301, 1387-1391 (2003). 
26. Jia, K. \& Levine, B. Autophagy is required for dietary restriction-mediated life span extension in C. elegans. Autophagy. 3, 597-9 (2007).

27. Hansen, M. et al. A role for autophagy in the extension of lifespan by dietary restriction in C. elegans. PLoS Genet. 4, e24 (2008).

28. Stead, E. R. et al. Agephagy - Adapting autophagy for health during aging. Front. Cell Dev. Biol. 7, 308 (2019).

29. McKay, J. P., Raizen, D. M., Gottschalk, A., Schafer, W. R. \& Avery, L. eat-2 and eat-18 are required for nicotinic neurotransmission in the Caenorhabditis elegans pharynx. Genetics. 166, 161-169 (2004).

30. Gomez-Amaro, R. L. et al. Measuring food intake and nutrient absorption in Caenorhabditis elegans. Genetics. 200, 443-454 (2015).

31. Brenner, S. The genetics of Caenorhabditis elegans. Genetics 77, 71-94 (1974).

32. Lionaki, E. \& Tavernarakis, N. High-throughput and longitudinal analysis of aging and senescent decline in Caenorhabditis elegans. Methods Mol. Biol. 965, 485-500 (2013).

33. Bishop, N. A. \& Guarente, L. Two neurons mediate diet-restriction-induced longevity in C. elegans. Nature. 447, 545-549 (2007).

34. Hinas, A., Wright, A. J. \& Hunter, C. P. SID-5 is an endosome-associated protein required for efficient systemic RNAi in C. elegans. Curr. Biol. 22, 1938-43 (2012).

35. Kamath, R. S., Martinez-Campos, M., Zipperlen, P., Fraser, A. G. \& Ahringer, J. Effectiveness of specific RNA-mediated interference through ingested double-stranded RNA in Caenorhabditis elegans. Genome Biol. 2, (2001).

36. R Core Team (2018). R: A language and environment for statistical computing. R 
Foundation for Statistical Computing, Vienna, Austria.URL https://www.Rproject.org/.

37. Bates Maechler, M., Bolker, B. \& Walker, A, D. lme4: Linear mixed-effects models using Eigen and S4. (2015).

38. Kuznetsova, A., Brockhoff, P. B. \& Christensen, R. H. B. lmerTest Package: Tests in Linear Mixed Effects Models . J. Stat. Softw. 82, (2017).

39. Ho, J., Tumkaya, T., Aryal, S., Choi, H. \& Claridge-Chang, A. Moving beyond P values: data analysis with estimation graphics. Nature Methods. 16, 565-566 (2019).

40. Therneau, T. M. Mixed Effects Cox Models [R package coxme version 2.2-14].

41. Schielzeth, H. Simple means to improve the interpretability of regression coefficients. Methods Ecol. Evol. 1, 103-113 (2010).

42. Lum, J. J., DeBerardinis, R. J. \& Thompson, C. B. Autophagy in metazoans: cell survival in the land of plenty. Nat. Rev. Mol. Cell Biol. 6, 439-48 (2005).

43. Gems, D. \& Partridge, L. Genetics of Longevity in Model Organisms: Debates and Paradigm Shifts. Annu. Rev. Physiol. 75, 621-644 (2013).

44. Partridge, L., Gems, D. \& Withers, D. J. Sex and death: what is the connection? Cell 120, 461-72 (2005).

45. Edwards, A. W. F. Human genetic diversity: Lewontin's fallacy. BioEssays. 25, 798801 (2003).

46. Flatt, T. Survival costs of reproduction in Drosophila. Exp. Gerontol. 46, 369-375 (2011).

47. Scott, R. C., Juhász, G. \& Neufeld, T. P. Direct Induction of Autophagy by Atg1 
48. Vellai, T., Bicsák, B., Tóth, M. L., Takács-Vellai, K. \& Kovács, A. L. Regulation of cell growth by autophagy. Autophagy. 4, 507-509 (2008).

49. Takacs-Vellai, K. et al. Inactivation of the autophagy Gene bec-1 triggers apoptotic cell death in C. elegans. Curr. Biol. 15, 1513-1517 (2005).

50. Hoffman, S., Martin, D., Meléndez, A. \& Bargonetti, J. C. elegans CEP-1/p53 and BEC-1 are involved in DNA repair. PloS one. 9, e88828 (2014).

51. Meléndez, A. \& Levine, B. Autophagy in C. elegans. Worm Book. 1-26 (2009). doi:10.1895/1.22.1

52. Palmisano, N. J. \& Meléndez, A. Autophagy in C. elegans development.

53. Ames, K. et al. A Non-Cell-Autonomous Role of BEC-1/BECN1/Beclin1 in Coordinating Cell-Cycle Progression and Stem Cell Proliferation during Germline

54. Williams, G. C. \& Williams, D. C. Natural selection of individually harmful social adaptations among sibs with special reference to social insects. Evolution. 11, 32-39

55. Maklakov, A. A. \& Chapman, T. Evolution of ageing as a tangle of trade-offs: energy versus function. Proc. R. Soc. B Biol. Sci. 286, 20191604 (2019).

56. de Magalhães, J. P. \& Church, G. M. Genomes Optimize Reproduction: Aging as a Consequence of the Developmental Program. Physiology. 20, 252-259 (2005). 

(2011).

515 58. Stergiou, L., Doukoumetzidis, K., Sendoel, A. \& Hengartner, M. O. The nucleotide excision repair pathway is required for UV-C-induced apoptosis in Caenorhabditis elegans. Cell Death Differ. 14, 1129-1138 (2007). 